\title{
An Estimation Theoretic Approach to the Asynchronous Detection of PSK Signals
}

\author{
Roberto Cristi, Sherif Michael \\ Department of Electrical and Computer Engineering \\ Naval Postgraduate School \\ Monterey, CA 93943-5100
}

\begin{abstract}
An algorithm is presented for smoothing data piecewise modeled by linear state space equations from measurements corrupted by additive noise. The main feature is the combination of a Markov Model with Kalman Filtering (KF) techniques in order to smooth and segment the data within the regions and detect the instant when the transitions occur. Particular attention is given to the filtering and segmentation of sinusoidal signals with periodic phase shifts and it is shown to operate as a PSK detector.
\end{abstract}

\section{Introduction}

The estimation of signals with time varying characteristics is of great interest in many areas of Signal Processing.

For this class of signals classical estimation techniques based on stationarity assumptions cannot be applied, and different techniques have to be investigated. For example signals with periodic characteristics can be analyzed by using the concept of cyclostationarity [1], [2], while a model based approach calls for estimation schemes based on stochastic state space models [3], [4].

In many instances of interest we can identify a class of nonstationary signals which can be modeled piecewise by deterministic state space equations with random initial conditions and measurement noise. This is the case, for example, of signals representable by Auto Regressive models with piecewise constant coefficients, of interest in speech processing and image processing [5].

For this class of signals we can approach the filtering and detection problems as a combination of a) segmentation into appropriate regions, and b) filtering within the regions themselves.

In this report we address the estimation problem of signals with piecewise state space models, and we present a suitable estimation algorithm. On a Maximum a Posteriori (MAP) framework we consider the compact regions as realizations of Markov models (as in related image processing approaches [6], [7]), and by combination with Kalman Filtering techniques a suitable likelihood function can be determined.

The estimation algorithm presented seems to be effective, and an extension to the multidimensional segmentation case has recently been developed [8].

Particular attention is given to PSK (Phase Shift Keying) signals, where the phase of a sinusoidal carrier is periodically shifted according to a multipolar modulating signal. In this particular case the recovery of the phase (and therefore the transmitted sequence) is based on the state space model that describes the sinusoid.
The report is divided in parts: the modeling assumption are stated in section 2, while the estimation problem and algorithm are given in sections 3 and 4 . Simulation results are given in section 5 .

\section{Statement of the Problem and Modeling Assumptions.}

The class of signals we consider are Phase Shift Keyed (PSK) sinusoids of the form

$$
y(\tau)=s(\tau)+w(\tau)
$$

with $s(\tau)=A_{M} \cos \left(\omega_{0} \tau+\phi(\tau)\right), \phi(\tau)$ being the phase information, piecewise constant in time, $\omega_{0}$ the carrier frequency and $w(\tau)$ the noise effect of channel, receiver and others. In particular we assume $w$ to be gaussian, white, zero mean for simplicity, with a known standard deviation $\sigma$.

The goal of this report is to recover the signal $s$ and the phase information $\phi$ from the noisy observations in an asynchronous fashion using estimation theoretic techniques based on Kalman Filter and Maximum a Posteriori estimation. The asynchronous detection implies that the receiver does not use any timing information from the transmitter.

On the basis of the assumptions we can model the signals $s, y$ piecewise by state equations, where the change on phase information is associated to change in the initial conditions from segment to segment.

For this purpose we define the processes $\gamma, x, y$ in the discrete time domain as follows

1. $\gamma=\{\gamma(t), t \in Z\}, \gamma(t) \in\{0,1\}$, the process of transitions , defined by the changing phase information as

$$
\gamma(t)= \begin{cases}1 & \text { if } \phi(t)=\phi(t-1) \\ 0 & \text { otherwise }\end{cases}
$$

2. $x=\{x(t), t \in Z\}$ and $y=\{y(t), t \in Z\}$, with $x \in R^{2}$, $y \in R$, defining the state and observation of the state space model

$$
\begin{cases}x(t+1) & =A x(t), \quad \text { if } \\ y(t) & =c x(t)+w(t)=0\end{cases}
$$

with initial conditions

$$
x\left(t_{k}\right)=\phi_{k}, \quad \text { if } \gamma\left(t_{k}\right)=1
$$

For the case of a sinusoid with frequency $\omega_{0}$ sampled at a rate $1 / T_{s}$ the matrices $A, c$ are given by 


$$
\begin{gathered}
A=\left[\begin{array}{cc}
\cos \theta_{0} & \sin \theta_{0} \\
-\sin \theta_{0} & \cos \theta_{0}
\end{array}\right] \\
c=[1,0]
\end{gathered}
$$

with $\theta_{0}=\omega_{0} T_{s}$ the digital frequency.

From simple trigonometric considerations it is easy to see that the phase information $\phi$ is contained in the initial conditions of the state space model (2.3) at the beginning of each block. Specifically from (2.3) we can write

$$
x_{1}(t)=A_{M} \cos \left(\theta_{0}\left(t-t_{k}\right)+\phi_{k}\right)
$$

for $t_{k} \leq t<t_{k+1}, t_{k}$ being the instants at which the phase changes, and

$$
\phi_{k}=t g^{-1} \frac{x_{2}\left(t_{k}\right)}{x_{1}\left(t_{k}\right)}
$$

On the process of transitions $\gamma$ we assume that an integer $d$ ex ists such that at most only one transition occurs in any interval $[t-d, t]$. Furthermore a $d$-Markov model of $\gamma$ is assumed, as

$$
\mathcal{P}(\gamma(t) \mid \gamma(t-1), \ldots \gamma(0))=P(\gamma(t) \mid \gamma(t-1), \ldots, \gamma(t-d-1))
$$

In order to assign an appropriate probability measure to $\gamma$ define the following sequences for all $t \in Z$ :

$$
\begin{aligned}
y_{t, d} & =[y(t), \ldots, y(t-d)] \\
\gamma_{t, d} & =[\gamma(t), \ldots, \gamma(t-d)] \\
y_{t} & =[y(t), \ldots, y(0)] \\
\gamma_{t} & =[\gamma(t), \ldots, \gamma(0)]
\end{aligned}
$$

and the vectors $\epsilon_{j}=\left[\epsilon_{j}(0), \ldots, \epsilon_{j}(d)\right] \in\{0,1\}^{d+1}$ as

$$
\epsilon_{j}(i)= \begin{cases}1 & \text { if } i=j \\ 0 & \text { if } i \neq j\end{cases}
$$

for $j=0, \ldots, d$. Also define $\epsilon_{-1}=0$. Then a probabilistic norlel for $\gamma$ is given by

$$
\mathcal{P}\left(\gamma(t)=0 \mid \gamma_{t-1, d}\right)= \begin{cases}1 & \text { if } \gamma_{t-1, d}=\epsilon_{j}, j \neq-1 \\ p_{0} & \text { if } \gamma_{t-1, d}=\epsilon_{-1}\end{cases}
$$

The reason behind this definition is the fact that $\gamma(t)=0$ with probability 1 if a transition exists within the interval $[t-d-$ $1, t-1$ ] (i.e. $\gamma_{t-1, d}=\epsilon_{j}, j \neq-1$ ). It there is no transition in the interval $[t-d-1, t-1]$ then $\gamma(t)=0$ with probability $p_{0}$ $\left(\right.$ and $\gamma(t)=1$ with probability $\left.p_{1}=1-p_{0}\right)$.

\section{Estimation.}

ln this section we determine an estimation scheme based on a Maximum a Posteriori probability approach (MAP) in order to extract the signal $x, \gamma$ from the observations $y$.

It is important to note the doubly stochastic nature of the process $x$. The doubly stochastic model comes from the fact that the signal can be described by a combination of two models: one for the regions, compact over $Z$, where the phase is constant, and one for the phase, which we assume random over each region. Within this framework, to estimate the signal implies an estimate of the regions of constant phase or, equivalently, of the transition points $t$ where $\gamma(t)=1$.

The algorithm we present is based on a moving window of length $d$ over which the likelihood of the transitions is recursively computed. In particular the following can be shown:

Lemma 1. For the process defined above the following recursion holds

$$
\begin{gathered}
\mathcal{P}\left(y_{t, d}, \gamma_{t, d} \mid y_{t-d-1}, \gamma_{t-d-1}\right)= \\
\frac{\mathcal{P}\left(y(t) \mid y_{t-1}, \gamma_{t}\right) \mathcal{P}\left(\gamma(t) \mid \gamma_{t-1}, y_{t-d-1}\right)}{\mathcal{P}\left(y(t-d-1) \mid y_{t-d-2}, \gamma_{t-d-1}\right) \mathcal{P}\left(\gamma(t-d-1) \mid y_{t-d-1}, \gamma_{t-d-2}\right)} \times \\
\times \mathcal{P}\left(y_{t-1, d}, \gamma_{t-1, d} \mid y_{t-d-2}, \gamma_{t-d-2},\right)
\end{gathered}
$$

Proof The result comes directly from Bayes factorization and it is shown in a forthcoming report [10].

By the recursion in lemma 1 we can update the statistics of $y$ and $\gamma$ over the interval $[t-d, t]$ conditional to past values of $y$ and $\gamma$. We can use this probability model to estimate the transitions at time $t-d$ on the basis of the observations up to time $t$. In particular we define

$$
\hat{\gamma}(t-d)=\operatorname{argmax}_{\gamma(t-d)} \mathcal{P}\left(\gamma(t-d) \mid y_{t}, \hat{\gamma}_{t-d-1}\right)
$$

By standard Bayesian arguments it is easy to see that the maximization (3.2) is equivalent to the maximization

$$
\hat{\gamma}(t-d)=\operatorname{argmax}_{\gamma(t-d)} \mathcal{P}\left(y_{t, d}, \gamma(t-d) \mid \hat{\gamma}_{t-d-1}, y_{t-d-1}\right)
$$

since

$$
\begin{aligned}
\mathcal{P}\left(y_{t, d}, \gamma(t-d) \mid \hat{\gamma}_{t-d-1} y_{t-d-1}\right)=\mathcal{P}\left(\gamma(t-d) \mid y_{t}, \hat{\gamma}_{t-d-1}\right) \times \\
\times \mathcal{P}\left(y_{t, d} \mid \hat{\gamma}_{t-d-1} y_{t-d-1}\right)
\end{aligned}
$$

and the rightmost term does not depend on $\gamma(t-d)$.

The likelihood on the right hand side of (3.3) can be recursively determined from the probability terms in lemma 1 . In particular, recalling that $\gamma_{t, d}$ only assumes realizations $\epsilon_{j}$, $j=-1, \ldots, d$ (since at most one transition occurs within any interval $[t-d, t])$ we can write

$$
\mathcal{P}\left(y_{t, d}, \gamma(t-d)=0 \mid \hat{\gamma}_{t-d-1}, y_{t-d-1}\right)=\sum_{j=-1}^{d-1} f_{t}\left(\epsilon_{j}\right)
$$

and

$$
\mathcal{P}\left(y_{t, d}, \gamma(t-d)=1 \mid \hat{\gamma}_{t-d-1}, y_{t-d-1}\right)=f_{t}\left(\epsilon_{d}\right)
$$

where we define

$$
f_{t}\left(\epsilon_{j}\right)=\mathcal{P}\left(y_{t, d}, \gamma_{t, d}=\epsilon_{j} \mid y_{t-d-1}, \hat{\gamma}_{t-d-1}\right)
$$

for $j=-1, \ldots, d$.

\section{Recursive Estimation}

On the above considerations it is shown in [10] that the process of transitions $\gamma$ can be estimated recursively as follows 


\section{Update:}

1. if $\hat{\gamma}_{t-d-1}=0$ then

$$
f_{t}\left(\epsilon_{j}\right)=C \mathcal{P}\left(\left.y(t)\right|_{t, d}=\epsilon_{j}, \hat{\gamma}_{t-d-1}, y_{t-1}\right) f_{t-1}\left(\epsilon_{j-1}\right),
$$

if $1 \leq j \leq d$,

$$
\begin{gathered}
f_{t}\left(\epsilon_{0}\right)=C \mathcal{P}\left(y(t) \mid \gamma_{t, d}=\epsilon_{j}, \hat{\gamma}_{t-d-1}, y_{t-1}\right) p_{1} f_{t-1}\left(\epsilon_{-1}\right) \\
f_{t}\left(\epsilon_{-1}\right)=C \mathcal{P}\left(y(t) \mid \gamma_{t, d}=\epsilon_{j}, \hat{\gamma}_{t-d-1}, y_{t-1}\right) p_{0} f_{t-1}\left(\epsilon_{-1}\right)
\end{gathered}
$$

with $C$ a constant independent of $\gamma_{t, d}$;

2. if $\hat{\gamma}_{t-d-1}=1$ then

$$
\begin{gathered}
f_{t}\left(\epsilon_{-1}\right)=C \mathcal{P}\left(y(t) \mid \gamma_{t, d}=\epsilon_{j}, \hat{\gamma}_{t-d-1}, y_{t-1}\right) f_{t-1}\left(\epsilon_{d}\right) \\
f_{t}\left(\epsilon_{k}\right)=0 \quad \text { if } k \neq-1
\end{gathered}
$$

Decision:

$$
\text { if } \sum_{j=-1}^{d-1} f_{t}\left(\epsilon_{j}\right)>f_{t}\left(\epsilon_{d}\right) \text { then } \hat{\gamma}_{t-d}=0 \text {, else } \hat{\gamma}_{t-d}=1 .
$$

The estimation and decision introduced above can be represented on the graph shown in figure 4.1. At each time $t$ the nodes marked as $-1, \ldots, d$ refer to the realizations of $\gamma_{t, d}$, and for each of these realizations the likelihood terms $f_{t}\left(\epsilon_{j}\right)$ are updated according to equations (4.1) and (4.2). The two branches converging to the node -1 are motivated by the fact that the realization $\gamma_{t, d}=\epsilon_{-1}$ (i.e. no transitions within the interral $[t-d, t])$ can come either from $\gamma_{t-1, d}=\epsilon_{-1}$ or $\gamma_{t-1, d}=\epsilon_{d}$.

The updating of the probability terms in (4.1), (4.2) can be determined by using well known properties of the Kalman Filter and the gaussian assumption. In particular at each node $j=-1, . ., d$ of the graph we update the estimate of $x$ and consequently the probability term as follows:

1. if $j=1, \ldots, d$,

$$
\begin{gathered}
\hat{x}(t \mid j)=A \hat{x}(t-1 \mid j-1)+K\left(P_{t}(j)\right)\left(y(t-1)-c^{T} \hat{x}(t-1 \mid j-1)\right) \\
P_{t}(j)=A P_{t-1}(j-1) A^{T}- \\
-K\left(P_{t-1}(j-1)\right)\left(c^{T} P_{t-1}(j-1) c+\sigma^{2}\right) K^{T}\left(P_{t-1}(j-1)\right)
\end{gathered}
$$

where

$$
K(P)=A P c\left(\sigma^{2}+c^{T} P c\right)^{-1}
$$

2. if $j=0$, then

$$
\begin{aligned}
\hat{x}(t \mid 0) & =x_{0} \\
P_{t}(0) & =P_{0}
\end{aligned}
$$

$x_{0}, P_{0}$ being initial conditions;

3. if $j=-1$ then $\hat{x}(t \mid-1)$ is updated as in (4.3), (4.4) with $j-1$ replaced by

$$
j-1-> \begin{cases}-1 & \text { if } \hat{\gamma}(t-1-d)=0 \\ d & \text { if } \hat{\gamma}(t-1-d)=1\end{cases}
$$

From the expressions of $\hat{x}(t \mid j)$ and $P_{t}(j)$ above we can determine the probability terms in $(4.1),(4.2)$, since they are gaussian with mean $c^{T} \hat{x}(t \mid j)$ and covariance $\sigma^{2}+c^{T} P_{t}(j) c$, i.e.

$$
\begin{gathered}
\ln \mathcal{P}\left(y(t) \mid \gamma_{t, d}=\epsilon_{j}, \hat{\gamma}_{t-d-1}, y_{t-1}\right)= \\
-1 / 2 \ln 2 \pi\left(\sigma^{2}+c^{T} P_{t}(j) c\right)-\frac{\left|y(t)-c^{T} \hat{x}(t \mid j)\right|^{2}}{2\left(\sigma^{2}+c^{T} P_{t}(j) c\right)}
\end{gathered}
$$

\section{Application Results and Comments.}

The algorithm presented in this paper, and still under development, has been applied to detection in several simulated examples. Although the general structure looks complicated at a first sight, the algorithm lends itself to a parallel structure implementation. Furthermore all the updatings of the probability terms in (4.1) - (4.9) can be implemented using precomputed terms since the values of the Kalman gains and covariance matrices are not data dependent.

In order to show the performances of the algorithm, several experiments on simulated data have been carried out. Fig. ure 4.1 shows the estimation of a PSK sinusoidal signal of amplitude 50 units, imbedded in an observation noise with standard deviation $\sigma=35$ units. The original, non noisy data is shown in figure 5.1 a), while the corrupted observations are given in figure $5.1 \mathrm{~b}$ ), and the result from the estimator in figure 5.1.c). It can be seen that the transitions are estimated by the algorithm.

\section{Conclusions.}

The problem of estimating a PSI signal is addresses in this report. Main feature of the algorithm devised is the fact that it is based on estimation theoretic considerartions, it is parallel and recursive in nature, and can be implemented using look up tables and precomputed gains. Furthermore the detection is completely asynchronous.

Since the algorithm is based on optimal estimation techniques, it is expected to be able to detect signals in the presence of a high level of noise. Further research is under way to determine the performance of the technique, its implementation and feasibility.

\section{REFERENCES}

[1] W. A. Gardner, " The Role of Spectral Correlation in Design and Performance Analysis of Synchronizers," IEEE Transactions on Communications, Vol COM 34, noll. November 1986;

[2] W. A. Gardner, Introduction to Random Processes with Applications to Signals and Systems, Macmillan. New Yorr.: 1985 ;

[3] G. Kitagawa, W. Gersh, "A Smoothness Priors Time Varying AR Coefficient Modeling of Nonstationary Covariance Time Series," IEEE Transactions on Automatic Control, Vol AC 30, January 1985; 
[1] W. Gersh, G. Kitagawa, "Smoothness Priors in Time Scrics," NPS Technical Report, Electrical and Computor" Eng. Dept., Naval Postgraduate School, Monterey, CA. April 1987;

[5] C. W. Therrien "An Estimation Theoretic Approach to Terrain Image Segmentation," Computer Vision, Graphics and Image Processing, Vol 22, June 1983;

[6] H. Derin, H. Elliott, "Modeling and Segmentation of Noisy and Textured Images Using Gibbs Random Fields," IEEE Transactions on Pattern Analysis and Machine Intelligence, Vol PAMI 9, January 1987;

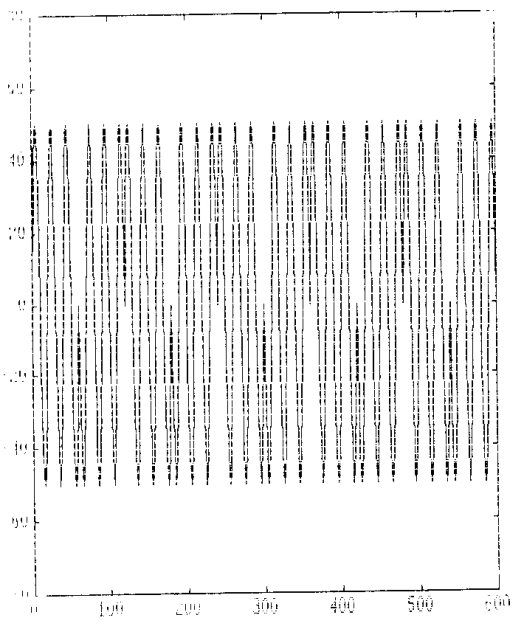

Fig. 5.1 a): original signal

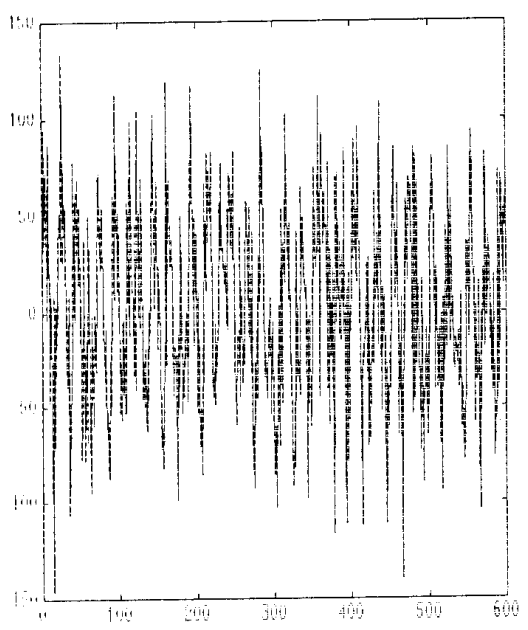

Fig. $5.1 \mathrm{~b})$ : noisy signal $($ sigma $=35.0)$
[7] H. Elliott, H. Derin, R. Cristi, D. Geman, "Application of the Gibbs Distribution to Image Segmentation," Proc. 1984 ICASSP, San Diego, CA, March 1984;

[8] R. Cristi, "Markov and Recursive Least Squares Methods for the Estimation of Data with Discontinuities," to appear in the IEEE Transactions on Acoustics Speech and Signal Processing, ;

[9] G.C. Goodwin, K.S. Sin, Adaptive Filtering Prediction and Control, Prentice Hall, 1984

[10] R. Cristi et al, "Detection of Signals with Discontinuities," in preparation.

t

$t+1$

$t+2$

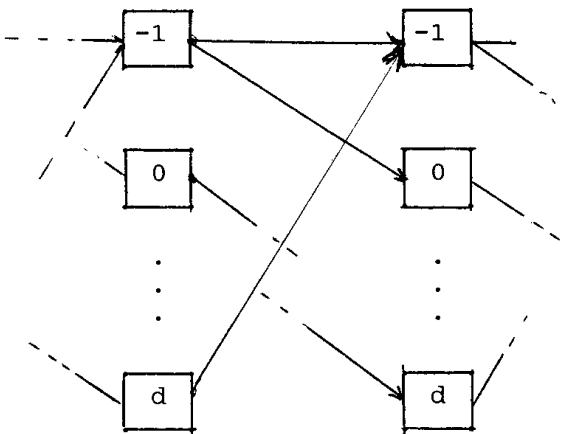

Figure 4.1: representation of the estimation algorithm.

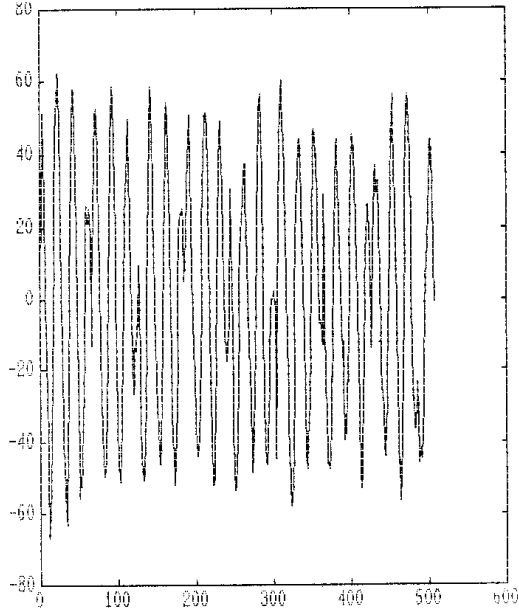

Fig. 5.1.c): estimated signal 